\title{
O espectro da reoperação em cirurgia de coronária
}

Jarbas J. DINKHUYSEN*, Luiz Carlos Bento de SOUZA*, Alberto M. ANIJAR**, Paulo P. PAULISTA**, Paulo CHACCUR ${ }^{\star *}$, Leopoldo S. PIEGAS*, Ricardo MANRIQUE*, Antoninho S. ARNONI***, J: Eduardo M. R. SOUSA*, Adib D. JATENE**

DINKHUYSEN, J. J.; SOUZA, L. C. B.; ANIJAR, A. M.; PAULISTA, P. P.; CHACCUR, P.; PIEGAS, L. S.; MANRIQUE, R.; ARNONI, A. S.; SOUSA, J. E. M. R.; JATENE, A. D. - O espectro da reoperação em cirurgia de coronária. Rev. Bras. Cir. Cardiovasc., 4(1): 32-42, 1989.

RESUMO: O presente trabalho tem por finalidade analisar os resultados cirúrgicos obtidos nos primeiros 30 dias de pós-operatório de um grupo de 204 pacientes submetidos a reintervenção coronária no Hospital do Coraçāo, entre janeiro de 1985 e setembro de 1988. O intervalo ocorrido entre a primeira e a segunda operação oscilou entre 1 mês e 16,5 anos (média $(7,3 \pm 3,7)$, sendo que, na maioria (120/58,8\%) dos casos, a indicação cirúrgica ocorreu em virtude de se constatar a progressão da doença aterosclerótica envolvendo os enxertos e a circulação nativa, enquanto que, em $71(34,8 \%)$ pacientes, observou-se apenas o comprometimento dos enxertos e, finalmente, em $13(6,4 \%)$ pacientes somente a circulação nativa apresentava lesōes ateroscleróticas obstrutivas. Cumpre salientar que um pequeno grupo $(9 / 4,4 \%)$ deste total de 204 casos sofreu uma terceira intervençāo, assim como em $20(9,8 \%)$ pacientes realizou-se, concomitantemente, correção cirúrgica de lesōes associadas, além da nova revascularização miocárdica. O perfil clínico desta série mostrou haver predomínio dos homens $(180 / 88 \%)$ e da faixa etária entre 50 e 64 anos, que englobou pouco menos que $2 / 3$ dos casos. A hipertensão (38\%) e a dislipemia $(26,5 \%)$ foram os fatores de risco mais presentes. A angina ocorreu em todos os casos, sendo a forma crônica instabilizada a mais freqüente $(99 / 48 \%)$. História de infarto do miocárdio ocorreu em $132(65 \%)$ pacientes, sendo a grande maioria (122/60\%) com evolução acima de 2 meses e $10(5 \%)$ com evolução recente. A angioplastia transluminal foi tentada, com sucesso, em $8(3,9 \%)$ pacientes. As principais diretrizes da técnica cirúrgica consistiram na heparinização (TCA entre 200 e $300 \mathrm{seg}$.) logo no início do procedimento, naqueles pacientes que apresentavam lesōes obstrutivas em pontes pérvias, isolamento do coração e vasos da base e das aderências pericárdicas, canulação de ambas as cavas, períodos intermitentes de anóxia miocárdica em hipotermia sistêmica moderada, utilização, sempre que possível, de uma ou ambas as mamárias, preponderantemente nos pacientes com idade inferior a 65 anos e tentar a revascularização completa. Foram realizados 547 enxertos coronários (2,7/pacientes), dos quais 311 (56,8\%) foram substituiçōes das pontes comprometidas e $236(42,1 \%)$ foram novos enxertos para artérias previamente não tratadas. A anastomose mamária-coronária foi empregada em $125(61 \%)$ pacientes, em configuração unilateral $102(50 \%)$ e bilateral $23(11 \%)$. Pequeno número de casos $(20 / 9,8 \%)$ sofreu procedimentos associados, na maioria aneurismectomia do ventrículo esquerdo (VE). Expressiva maioria desta série (127/62,5\%) evoluiu rotineiramente; contudo, $58(28,4 \%)$ pacientes apresentaram algum tipo de complicação não fatal, com resposta favorável à terapêutica especifica. Ocorreram $19(9,3 \%)$ óbitos. Analisamos as influências que os diversos fatores causaram nos resultados e concluímos que: 1) pacientes com lesōes apenas na circulação nativa apresentaram maior morbidade ( $P=0,70$ ), enquanto que, naqueles com lesōes nos enxertos e na circulação nativa, a mortalidade foi mais alta $(P=0,82) ; 2)$ a idade dos pacientes influiu inversamente à expectativa, pois o grupo mais jovem (35 a 49 anos) mostrou maior mortalidade $(P=0,93)$, enquanto que, nos pacientes com idade acima de 65 anos, ocorreu maior incidência de complicaçōes não fatais $(P=0,18) ; 3)$ o emprego das artérias mamárias na reintervenção nāo pressupôs acréscimo de complicaçōes ou mortalidade $(P>0,05)$; 4) a correçāo cirúrgica concomitante de lesão associada representou prognóstico mais severo com indices significativos de morbidade $(P=0,38)$ e de mortalidade $(P=0,02)$, este último dado estatisticamente significativo; 5) o infarto recente do miocárdio também influiu decisivamente; contudo, este subgrupo apresentou maior morbidade ( $P=0,80$ ) do que mortalidade $(P=0,57) ; 6$ ) finalmente, na 3 : intervenção, angioplastia mal sucedida e presença de mamária prévia não causaram impacto decisivo na morbidade e na mortalidade desta série ( $P>0,05)$.

DESCRITORES: miocárdio, revascularização, reoperação.

\footnotetext{
Trabalho realizado no Hospital do Coraçăo da Associaçăo do Sanatório Sírio e no Instituto Dante Pazzanese de Cardiologia. São Paulo, SP, Brasil.

* Do Hospital do Coração da Associação do Sanatório Sírio e do Instituto Dante Pazzanese de Cardiologia.

** Do Hospital do Coração da Associação do Sanatório Sírio.

*** Do Instituto Dante Pazzanese de Cardiologia.

Endereço para separatas: Jarbas Dinkhuysen. Rua Desembargador Eliseu Guilherme, 123. 04004 Paraiso, Săo Paulo, SP, Brasil.
} 
DINKHUYSEN, J. J.; SOUZA, L. C. B.; ANIJAR, A. M.; PAULISTA, P. P.; CHACCUR, P.; PIEGAS, L. S.; MANRIQUE, R.; ARNONI, A. S.; SOUSA, J. E. M. R.; JATENE, A. D. - O espectro da reoperação em cirurgia de coronária. Rev. Bras. Cir. Cardiovasc., 4(1): 32-42, 1989.

\section{INTRODUÇÃO}

A revascularização cirúrgica do miocárdio é um método consagrado no tratamento da aterosclerose coronária obstrutiva, pois, através dele, se consegue a eliminação, ou mesmo a redução dos sintomas em 85 a $90 \%$ dos $\operatorname{casos}^{19}$. A despeito dos progressos terapêuticos clínico-cirúrgicos conseguidos, a doença mantém caráter evolutivo, numa boa parte dos pacientes, tendo sido relatada a recurrência dos sintomas em $3,5 \%$ a $7,2 \%$ dos pacientes, a cada ano de evolução pós-operatória a longo prazo ${ }^{4.5}$.

O retorno da angina geralmente é conseqüência do comprometimento aterosclerótico das pontes de safena, das artérias coronárias tratadas ou não, ou da combinação destes fatores, possibilitando-se, assim, a necessidade de uma nova operação.

O número destas reivindicaçōes tem crescido e as projeções futuras indicam que a tendência é aumentar ainda mais ${ }^{2}$. Em nosso meio, este crescimento também ocorre, porque o número de intervençōes nas coronárias tem crescido significativamente.

Inúmeras publicações demonstram os resultados das reoperaçōes ${ }^{3}, 11,16,18,20,23$ e são unânimes em concluir que, a despeito da melhoria dos diferentes métodos de proteção miocárdica e da experiência cirúrgica, os riscos ainda superam os da primeira intervenção ${ }^{3}$. É, sem dúvida, um procedimento cheio de peculiaridades, que difere substancialmente da primeira intervenção e no qual inúmeros fatores jogam papel decisivo e, se não forem convenientemente valorizados, as possibilidades de insucesso aumentam.

Este trabalho tem por finalidade a análise do procedimento, nos primeiros 30 dias, e dos fatores que influenciaram os resultados, nesta fase.

\section{CASUÍSTICA E MÉTODOS}

Trata-se de um grupo consecutivo de 204 pacientes, operados entre janeiro de 1985 e setembro de 1988. Todos os dados relativos aos primeiros dias, incluindo história clínica, cirurgia, pós-operatório imediato e alta hospitalar, foram obtidos com auxílio de computador alimentado com dados clínico-cirúrgicos registrados em ficha específica individual. A análise estatística foi feita com o teste $\boldsymbol{x}^{2}$.

\section{PACIENTES}

São 204 pacientes, sendo $180(88 \%)$ do sexo masculino e $24(12 \%)$ do feminino, com idade entre $34 \mathrm{e}$ 77 anos $(57,5 \pm 9,2)$. Na faixa entre 30 e 45 anos, estavam $26(12,7 \%)$ pacientes; entre 46 e 60 anos, 99
$(48,5 \%)$ pacientes; entre 61 e 69 anos, $64(31,2 \%)$ pacientes e acima de 70 anos, $15(7,5 \%)$ pacientes.

Os antecedentes pessoais isolados foram hipertensão arterial em 79 (38\%) pacientes, dislipemia em 54 $(26,5 \%)$, diabete em $19(4,5 \%)$ e sem antecedentes clínicos em $62(31 \%)$. Infarto do miocárdio prévio à reintervenção ocorreu em $132(65 \%)$, sendo que a maioria $(122 / 60 \%)$ tinha evolução acima de 2 meses e $10(5 \%)$ com evolução abaixo de 2 meses.

Praticamente todos os pacientes apresentavam manifestação clínica de angina, sendo a forma crônica instabilizada a mais freqüente, tendo ocorrido em $99(48,5 \%)$ pacientes, seguida de angina crônica estável $(52 / 25,5 \%)$. As formas instável $(20 / 9,8 \%)$, a de recente começo $(20 / 9,8 \%)$, a síndrome intermediária $(7 / 3,5 \%)$, após infarto agudo do miocárdio (IAM) $(4 / 1,9 \%)$ e a isquemia silenciosa $(2 / 0,9 \%)$ foram as menos freqüentes.

A classe funcional para angina foi categorizada, conforme a classificação da New York Heart Association (NYHA), observando-se que a maioria dos pacientes se encontrava nas classes III e IV (Tabela 1).

O passado mórbido desta série foi bem significativo, pois $91(44,6 \%)$ pacientes referiram antecedentes variados, destacando-se os gastroduodenais $(21 / 23 \%)$, renais $(16 / 17,5 \%)$, pulmonares $(11 / 12 \%)$, hepáticos $(8 / 8,8 \%)$ e outros menos freqüentes, chamando-se a atenção para um grupo de $8(8,8 \%)$ pacientes que se submeteram a angioplastia prévia.

O intervalo transcorrido entre a primeira e a segunda operaçāo oscilou entre 1 mês a 16,5 anos $(7,3 \pm 3,7)$, sendo que, na maioria dos pacientes $(120 / 58,8 \%)$, foram observadas progressão da doença envolvendo ambos os enxertos e circulação nativa; em $71(34,8 \%)$ apenas os enxertos estavam comprometidos e, em $13(6,4 \%)$, somente a circulação coronária nativa (Tabela 2).

TABELA 1

CLASSE FUNCIONAL (NYHA)

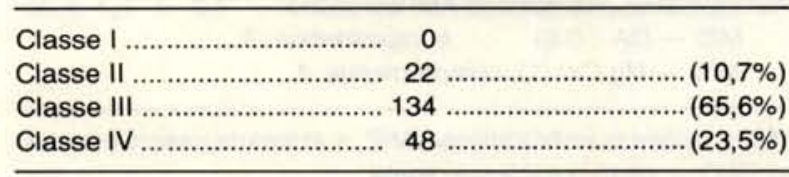

TABELA 2

PROGRESSĀO DA DOENCAA NOS ENXERTOS E CIRCULAÇAO NATIVA

\footnotetext{
Intervalo 1: e 2: operação - 1 mês a 16,5 anos $(7,3 \pm 3,7)$ Comprometimento:
- enxerto e circulação nativa ............... $120 \quad(58,8 \%)$
- somente nos enxertos ....................... $71 \quad(34,8 \%)$

- somente na circulação nativa ............ $13 \quad(6,4 \%)$
} 
DINKHUYSEN, J. J.; SOUZA, L. C. B.; ANIJAR, A. M.; PAULISTA, P. P.; CHACCUR, P.; PIEGAS, L. S.; MANRIQUE, R.; ARNONI, A. S.; SOUSA, J. E. M. R.; JATENE, A. D. - O espectro da reoperação em cirurgia de coronária. Rev. Bras. Cir. Cardiovasc., 4(1): 32-42, 1989.

Nesta série de pacientes, contrariamente à expectativa, os enxertos mostraram ligeira tendência em manter a perviabilidade por maior tempo $(7,1 \pm 3,5$ anos) do que a circulaçăo coronária nativa, cujo tempo de evoluçāo foi $6,9 \pm 3,9$ anos. Outro aspecto que chamou a atenção foi que, de 17 anastomoses mamária-coronária, 8 estavam obstruídas e o tempo médio de perviabilidade deste subgrupo específico foi $4,2 \pm 5,1$ anos (Tabela 3$)$.

Contudo, é necessário ressaltar que a perviabilidade das anastomoses mamária/coronária (4,2 $\pm 5,1$ anos) foi inferior à dos enxertos $(7,1 \pm 3,5$ anos) e da circulaçāo nativa (6,9 $\pm 3,9$ anos), tendo em vista que a aplicaçāo desta técnica, em nosso Serviço, foi iniciada em 1972 e praticamente interrompida em 1974, sendo vigorosamente reativada em $1983^{7}$. Por este motivo, a comparação de perviabilidade entre estes grupos fica prejudicada.

Um pequeno grupo de pacientes $(20 / 9,8 \%$ ) apresentava lesōes associadas passíveis de tratamento cirúrgico concomitante, havendo predomínio de aneurisma do ventrículo esquerdo (VE). Outros 9 (4,4\%) pacientes já haviam sido submetidos a reintervenção, sendo, portanto, candidatos à 3 : operação.

Pequeno número de pacientes $(20 / 9,8 \%)$ sofreu procedimentos associados, sendo a maioria aneurismectomia de VE e correçōes orovalvares (Tabela 4).

\section{RESULTADOS}

Expressiva maioria desta série (127/62,5\%) evoluiu rotineiramente e $58(28,4 \%)$ pacientes apresentaram algum tipo de complicação não fatal com resposta favorável à terapêutica específica. Ocorreram 19 (9,3\%) óbitos.

TABELA 3

PERVIABILIDADE DOS ENXERTOS E CIRCULAÇÃO NATIVA

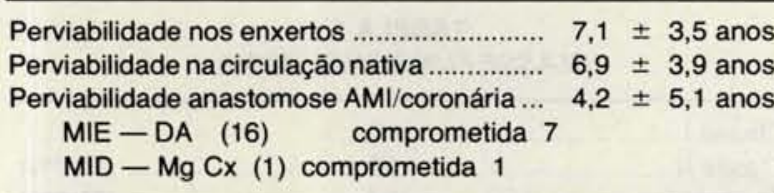

$\mathrm{AMI}=$ artéria mamária interna; $\mathrm{MIE}=$ mamária interna esquerda; MID = mamária interna direita

TABELA 4

\section{PROCEDIMENTOS ASSOCIADOS}

\begin{tabular}{|c|c|c|}
\hline Aneurismectomia .................................................. & 14 & $(6,8 \%)$ \\
\hline Comissurotomia aórtica ...................................... & 2 & $(1,0 \%)$ \\
\hline Plastia mitral ......................... & 1 & \\
\hline 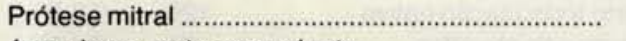 & 1 & \\
\hline Aneurisma aorta ascendente ................................ & 1 & \\
\hline Endarterectomia carótida .................................... & 1 & \\
\hline
\end{tabular}

As complicaçōes, concomitantes ou não, estão relacionadas conforme sua ocorrência nas diferentes fases da internaçāo, a fim de que se possa ter mais elementos de análise. No centro cirúrgico, as ocorrências mais freqüentes foram a suspeita de IAM em $9(4,4 \%)$ pacientes, sídrome de baixo débito em $8(3,9 \%)$, arritmias em 6 $(2,9 \%)$, parada cardiaca em $3(1,4 \%)$ e outros. Ocorreram $4(1,9 \%)$ óbitos, nesta fase.

\section{TÉCNICA CIRÚRGICA}

Todos os pacientes foram operados através de toracotomia mediana, com liberação das aderências pericárdicas, de maneira a isolar completamente as câmaras cardiacas e os grandes vasos, evitando-se manipulaçōes nas pontes de safena comprometidas. No início da toracotomia, os pacientes eram heparinizados em dose suficiente para manter o tempo de coagulação ativada (TCA) entre 200 e 300 segundos, mormente naqueles que tinham lesōes semi-obstrutivas nas pontes de safena. A instalação da circulação extracorpórea era feita pela canulação da aorta ascendente e de ambas as cavas. As anastomoses distais e proximais eram feitas em paradas anóxicas intermitentes com hipotermia sistêmica moderada $\left(32^{\circ} \mathrm{C}\right)$. Na maioria dos pacientes, a artéria mamária interna esquerda foi usada e, sempre que possível, aplicando-se ambas as mamárias, preponderantemente nos pacientes com idade inferior a 65 anos, completando-se com pontes de safena, de maneira a realizar a revascularização completa do miocárdio.

Foram realizados 547 enxertos coronários (média 2,7 pontes/paciente), dos quais $311(56,8 \%)$ substituiçōes das pontes comprometidas e $236(42,1 \%)$ foram enxertos para artérias não tratadas. $\mathrm{A}$ anastomose mamária-coronária foi empregada em $125(61 \%)$ pacientes, em configuração unilateral geralmente MIE-DA em 102 $(50 \%)$ pacientes e bilateral em $23(11 \%)$ (Tabela 5$)$.

No centro cirúrgico, ocorreu suspeita de IAM em $9(4,4 \%)$ casos, síndrome de baixo débito em $8(3,9 \%)$, parada cardiaca em $3(1,4 \%)$, acidentes cirúrgicos relacionados com: veia inominada 1, átrio esquerdo 1 e com a aorta 1 por rotura de aneurisma. Dois pacientes não

TABELA 5 PROCEDIMENTOS ASSOCIADOS

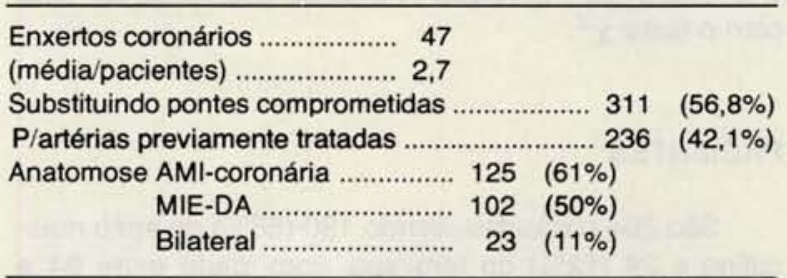

$\mathrm{AMI}=$ artéria mamária interna; MIE = mamária interna esquerda; $\mathrm{DA}=$ descendente anterior. 
DINKHUYSEN, J. J.; SOUZA, L. C. B.; ANIJAR, A. M.; PAULISTA, P. P.; CHACCUR, P.; PIEGAS, L. S.; MANRIQUE, R.; ARNONI, A. S.; SOUSA, J. E. M. R.; JATENE, A. D. - O espectro da reoperação em cirurgia de coronária. Rev. Bras. Cir. Cardiovasc., 4(1): 32-42, 1989.

saíram de circulação extracorpórea e houve necessidade de laparotomia exploradora em 1 caso. Constataram-se $4(1,9 \%)$ óbitos na sala de operaçōes.

$\mathrm{Na}$ unidade de terapja intensiva, já na fase de recuperação pós-operatória imediata, verificou-se que complicaçōes respiratórias $(22 / 10,7 \%)$, arritmias (19/9,3\%), complicaçōes neurológicas $(15 / 7,3 \%)$, síndrome de baixo débito $e$ insuficiência cardíaca $(10 / 4,9 \%)$ foram as mais freqüentes. Constataram-se $14(6,8 \%)$ óbitos, nesta fase da internação.

$\mathrm{Na}$ enfermaria, observaram-se complicaçōes com menor freqüência e gravidade, sendo secreção esternal $(8 / 3,9 \%)$, fibrilação atrial $(6 / 2,9 \%)$ e derrame pleural $(4 / 1,9 \%)$ as que mais ocorreram.

O tempo de internação hospitalar oscilou entre 8 e 60 dias $(12,6 \pm 6)$.

Com o intuito de analisar mais detalhadamente a influência das diversas variáveis sobre os resultados, procuramos verificar, em cada variável (subgrupo), a incidência de morbidade e mortalidade e comprovar seus resultados. Deste modo, em primeiro lugar relacionamos, como auxílio da cinecoronariografia, os subgrupos de pacientes com lesão nos enxertos e circulação nativa, com lesão apenas nos enxertos e, por último, com lesão apenas na circulaçāo nativa.

Observa-se ocorrência de maior morbidade no subgrupo de pacientes com lesōes apenas na circulação nativa $(38,5 \%)$, enquanto que, por outro lado, a mortalidade foi mais incidente no subgrupo de pacientes que apresentavam lesão, tanto nos enxertos, quanto na circu- lação nativa (10\%), ambos sem significância estatística $(P>0,05)$ (Tabela 6).

Em segundo lugar, estão os subgrupos formados, respectivamente, pelos pacientes que receberam anastomose mamária-coronária na reintervenção, portadores de lesões associadas tratados, simultâneamente, com anastomose mamária-coronária prévia, com infarto do miocárdio de evolução recente (abaixo de 2 meses) submetidos a 3: intervenção e, finalmente, os submetidos previamente a angioplastia.

Chama a atenção que o emprego da artéria mamária na reintervençāo nāo constitui fator significativo, tanto na morbidade, quanto na mortalidade, nesta série. O tratamento concomitante de lesōes associadas foi fator que influenciou nos resultados, principalmente na mortalidade (35\%), sendo este valor significativamente estatístico ( $P>0,05 \%$ ). O infarto recente do miocárdio com evolução inferior a 2 meses, a 3a intervenção e a angioplastia prévia também influíram na morbidade, contudo sem significância estatística. A presença de anastomose mamária-coronária prévia à reoperação nāo constituiu fator importante que influísse nos resultados (Tabela 7).

Por último, dividimos os pacientes em grupos etários distintos, com idade variando de 35 a 49 anos, 50 a 64 anos e acima de 65 anos.

Observou-se que o subgrupo mais idoso (acima de 65 anos), apresentou maior índice de morbidade (39\%) e, paradoxalmente, a menor mortalidade $(4,1 \%)$, enquanto que o mais jovem (35-49 anos) mostrou menor morbidade $(22,5 \%)$ e a maior mortalidade $(11,5 \%)$ (Tabela 8$)$.

TABELA 6

MORBIDADEMORTALIDADE SUBGRUPOS ANGIOGRAFICOS

\begin{tabular}{|c|c|c|c|c|c|c|c|}
\hline \multirow[b]{2}{*}{ Lesão enxertos e circulação nativa } & \multirow{2}{*}{$\frac{N \cdot}{120}$} & \multicolumn{2}{|c|}{$M O R B I D A D E$} & \multirow{2}{*}{$\frac{P\left(X^{2}\right)}{0,98}$} & \multicolumn{2}{|c|}{ MORTALIDADE } & \multirow{2}{*}{$\frac{P\left(X^{2}\right)}{0,99}$} \\
\hline & & 35 & $(29 \%)$ & & 12 & $(10 \%)$ & \\
\hline Lesāo apenas nos enxertos & 71 & 18 & $(25,3 \%)$ & 0,72 & 6 & $(8,5 \%)$ & 0,98 \\
\hline Lesāo apenas na circulação nativa & 13 & 5 & $(38,5 \%)$ & 0,64 & 1 & $(7,7 \%)$ & 0,76 \\
\hline
\end{tabular}

TABELA 7

MORBIDADEMORTALIDADE SUBGRUPOS DIVERSOS

\begin{tabular}{|c|c|c|c|c|c|c|c|}
\hline \multirow[b]{2}{*}{ Emprego da mamária } & \multirow{2}{*}{$\frac{N:}{120}$} & \multicolumn{2}{|c|}{ MOABIDADE } & \multirow{2}{*}{$\frac{P\left(X^{2}\right)}{0,84}$} & \multicolumn{2}{|c|}{ MORTALIDADE } & \multirow{2}{*}{$\frac{P\left(X^{2}\right)}{0,46}$} \\
\hline & & 23 & $(19,1 \%)$ & & 8 & $(06,6 \%)$ & \\
\hline Patologias associadas & 20 & 4 & $(20,0 \%)$ & 0,38 & 7 & $(35,0 \%)$ & 0,02 \\
\hline Mamária prévia & 17 & 2 & $(11,7 \%)$ & 0,22 & & zero & 0,38 \\
\hline IAM recente & 10 & 3 & $(30,0 \%)$ & 0,80 & 2 & $(20,0 \%)$ & 0,57 \\
\hline 3: intervenção & 9 & 2 & $(22,0 \%)$ & 0,97 & 1 & $(11,0 \%)$ & 0,68 \\
\hline Angioplastia prévia & 8 & 2 & $(25,0 \%)$ & 0,85 & 1 & $(12,5 \%)$ & 0,75 \\
\hline
\end{tabular}

$I A M=$ infarto agudo do miocárdio. 
DINKHUYSEN, J. J.; SOUZA, L. C. B.; ANIJAR, A. M.; PAULISTA, P. P.; CHACCUR, P.; PIEGAS, L. S.; MANRIQUE, R.; ARNONI, A. S.; SOUSA, J. E. M. R.; JATENE, A. D. - O espectro da reoperaçāo em cirurgia de coronária. Rev. Bras. Cir. Cardiovasc., 4(1): 32-42, 1989.

TABELA 8

MORBIDADEMORTALIDADE DOS SUBGRUPOS ETARIOS

\begin{tabular}{|c|c|c|c|c|c|c|c|}
\hline \multirow[b]{2}{*}{ Idade $35-49$ anos } & \multirow{2}{*}{$\frac{N:}{35}$} & \multicolumn{2}{|c|}{ MORBIDADE } & \multirow{2}{*}{$\frac{P\left(X^{2}\right)}{0,63}$} & \multicolumn{2}{|c|}{ MORTALIDADE } & \multirow{2}{*}{$\frac{P\left(X^{2}\right)}{0,93}$} \\
\hline & & 8 & $(22,5 \%)$ & & 4 & $(11,5 \%)$ & \\
\hline Idade $50-64$ anos & 121 & 31 & $(26,6 \%)$ & 0,67 & 13 & $(10,7 \%)$ & 0,82 \\
\hline Idade acima de 65 anos & 48 & 19 & $(39,0 \%)$ & 0,18 & 2 & $(4,1 \%)$ & 0,38 \\
\hline
\end{tabular}

\section{DISCUSSÃO}

Inúmeros trabalhos têm mostrado resultados cirúrgicos satisfatórios na reintervençāo para revascularizaçāo do miocárdio, com mortalidade oscilante entre 1,3\% a $12,5 \%{ }^{2}, 21$. Nas duas maiores séries publicadas, a mortalidade oscilou entre 4,0 e $4,8 \%$ nos primeiros 30 dias $^{15}$. 22. Apesar do risco cirúrgico mais elevado que o procedimento primário, a mortalidade na reoperação parece razoavelmente baixa, se considerarmos que os pacientes, freqüentemente, apresentam graus mais avançados de aterosclerose coronária e o procedimento ser tecnicamente mais difícil ${ }^{24}$.

A presente série de 204 pacientes consecutivos, que foram para reintervenção, apresentou características pré-operatórias com importantes componentes de antecedentes pessoais e passado mórbido, sinais clínicos evidentes de comprometimento aterosclerótico das artérias coronárias nativas e dos enxertos. A classe funcional (NYHA) para angina categorizou a maioria dos pacientes nas classes III e IV, fazendo pressupor estarmos analisando um grupo de prognóstico cirúrgico mais reservado.

O intervalo de tempo entre a 1: e a 2a intervenção não foi particularmente diferente da literatura e, como se podia prever, apesar de manter a perviabilidade por maior tempo que a circulação nativa, os enxertos foram mais afetados pela aterosclerose. A perviabilidade da anastomose AMI-coronária foi menor que as pontes de safena; contudo, deve-se ressaltar que esta comparação não pode ser feita tendo em vista o pequeno número de mamárias utilizado e, conforme já foi citado ${ }^{7}$, sua aplicação foi descontinuada por longo período, no nosso Serviço.

$\mathrm{Na}$ revisão bibliográfica realizada, não havia citações sobre casos que foram para reoperações coronárias e tratamento de lesōes associadas simultaneamente; nesta série os casos que apresentavam lesōes associadas tiveram a cirurgia cororiária como principal indicação, sendo que a maioria apresentava aneurisma na evolução tardia da primeira intervenção. Outro subgrupo incluido nesta série foi de pacientes que, pela 3 : vez, se submetiam a revascularização do miocárdio.

Os principais pontos da técnica cirúrgica empregada se firmaram na abertura do tórax por toracotomia media- na e liberação completa do coração e vasos da base das aderências pericárdicas, o que impossibilitou a abordagem confortável de toda a rede arterial coronária. Contudo, alguns autores ${ }^{3}{ }^{25}$ demonstraram que, em casos selecionados nos quais a artéria circunflexa e seus ramos necessitam de revascularizaçăo, a abordagem por toracotomia esquerda mostrou utilidade. Outro aspecto é a anticoagulação com heparina (TCA entre 200 e 300 segundos) na fase da abertura do tórax, cuja finalidade é evitar eventuais estados de hiperagregabilidade ${ }^{18}$, que poderiam agravar lesōes críticas, tanto nos enxertos, quanto na circulação nativa. A ocorrência de quadros de isquemia aguda e infarto no transoperatório das reintervenções tem sido relatada praticamente em todos os trabalhos. Alguns autores ${ }^{10,14,26}$ chamam a atenção para este fato e identificam a embolia distal de material trombo-aterosclerótico na ponte de safena como uma das causas mais freqüentes desses episódios. O ateroma, nessas pontes, é freqüentemente difuso e friável, desprendendo-se com facilidade e embolizando perifericamente, levando a isquemia aguda e insuficiência ventricular aguda ${ }^{9}$.

Têm sido relatadas diferentes técnicas cirúrgicas para evitar esse tipo de complicação ${ }^{10,23}$, que consistem na exposição do átrio direito e aorta o suficiente para canulação e instalação da circulação extracorpórea e, em hipotermia, liberar as aderências ligando proximal e distalmente as pontes comprometidas, evitando, assim, embolias periféricas. Todavia, esta manobra não é isenta de complicaçōes, podendo provocar quadros isquêmicos com insuficiência ventricular, principalmente se as pontes forem ainda pérvias.

A proteção miocárdica foi obtida através de paradas anóxicas curtas intermitentes, relativas a cada anastomose, em hipotermia sistêmica moderada $\left(32^{\circ} \mathrm{C}\right)$. A grande maioria dos grupos cirúrgicos se utiliza da parada miocárdica induzida e mantida com cardioplegia a $4^{\circ} \mathrm{C}$. Nestas condiçōes, é necessário levar-se em conta que a infusão de solução cristalóide de cardioplegia pode ser argüída como causa determinante de embolização periférica, devido a níveis baixos de densidade, viscosidade e temperatura ${ }^{10}$, que predisporiam deslocamentos de material aterosclerótico na ponte de safena comprometida. Nesta eventualidade, este autor ${ }^{10}$ sugere a infusão de cardioplegia por via retrógrada, via seio coronário. 
DINKHUYSEN, J. J.; SOUZA, L. C. B.; ANIJAR, A. M.; PAULISTA, P. P.; CHACCUR, P.; PIEGAS, L. S.; MANRIQUE, R.; ARNONI, A. S.; SOUSA, J. E. M. R.; JATENE, A. D. - O espectro da reoperaçăo em cirurgia de coronária. Rev. Bras. Cir. Cardiovasc., 4(1): 32-42, 1989.

As veias safenas utilizadas nas pontes aorto-coronárias foram obtidas, geralmente, na perna preservada na primeira operação; contudo, em alguns casos, devido à impossibilidade de utilização, ou mesmo pela sua ausência, foram empregadas veias dos membros superiores. O uso das artérias mamárias foi amplamente perseguido, na medida de sua viabilidade e indicação a cada caso. COSGROVE et alii ${ }^{6}$ concluíram que um dos fatores preditivos de risco de reintervenção é a ausência de anastomose AMl-coronária na primeira intervenção, devido às suas características de perviabilidade a longo prazo. Em nossa série, $61 \%$ dos pacientes receberam anastomose mamária-coronária em configuração unilateral e bilateral, preponderantemente nos pacientes com idade inferior a 65 anos.

Um dos aspectos técnicos que deve ser considerado é a necessidade de ressecção completa da ponte de safena comprometida pela aterosclerose, porque sua contigüidade com o leito arterial, a despeito da interposição mais distal de um novo enxerto, pode predispor à embolização do material tromboaterosclerótico, principalmente naqueles que não apresentam obstrução importante na cinecoronariografia, mas, durante o ato cirúrgico, palpam-se endurecimentos, no seu trajeto, denotando presença de placas de ateroma, nas paredes. De um modo geral, a conduta adotada tem sido, também, a sua substituição ${ }^{10}$ por enxertos novos, evitando, assim, problemas obstrutivos futuros, principalmente nos pacientes jovens.

De um modo geral, procurou-se realizar a revascularizaçăo completa (2,8 enxertos por paciente), incluíndo artérias previamente afetadas que funcionariam como eventual ponte de circulação colateral futura. A revascularização completa ${ }^{12} \mathrm{e}$ uso de artérias mamárias ${ }^{6}$ constituem-se nas únicas variáveis que influem, decisivamente, nos resultados tardios da revascularização cirúrgica do miocárdio.

Os índices de morbidade e mortalidade obtidos nesta série se mostraram mais elevados quando comparados com outras séries $1,6,9,10,12,15,16,24$. Contudo, é necessário ressaltar que aqui estāo incluídos subgrupos de pacientes com lesōes associadas, com infarto recente do miocárdio, de 3 : intervenção, que, obviamente, tornam o prognóstico mais grave.

A presença de pontes de safena comprometidas, tanto no subgrupo angiográfico de lesāo no enxerto e circulação nativa, assim como no subgrupo de lesão apenas nos enxertos, apesar de não haver significância estatística ( $P>0,05$ ), foi fator que determinou maior morbi-mortalidade. Reintervençāo em pacientes com enxertos pérvios e lesōes obstrutivas apenas na circulação nativa podem representar prognóstico melhor, semeIhante à primeira intervençăo.

É unânime o conceito de que o uso da artéria mamária em cirurgia de revascularizaçāo constitui fator deci- sivo na obtenção de bons resultados a longo prazo. Nas reintervençōes, este conceito se reveste ainda de maior importância, visto que este grupo de pacientes é, inequivocamente, portador de aterosclerose insidiosa e de difícil controle. Por conseguinte, o uso da anastomose AMIcoronária deve fazer parte obrigatória da planificaçāo cirúrgica das reintervenções, principalmente em pacientes com idade inferior a 60 anos, exceto nos casos de absoluta impossibilidade. Esta modalidade técnica não representou acréscimo de morbi-mortalidade do grupo em estudo, quando comparado com os obtidos na primeira operação ${ }^{7}$.

A presença concomitante de lesōes associadas, nas reoperaçōes coronárias, empobrece o prognóstico cirúrgico de forma significativa. Somam-se os riscos de reoperação e da lesão associada pela maior manipulação cirúrgica, pelo aumento do tempo de circulação extracorpórea e pelos inúmeros outros fatores, resultando em maiores probabilidades de complicaçōes. Este fato ficou bem evidenciado nos resultados cirúrgicos deste subgrupo, o qual mostrou mortalidade elevada estatisticamente significativa $(P>0,05)$.

Outro fator importante de risco, nas reoperaçōes, é a disfunção ventricular esquerda ${ }^{7}$. Aproximadamente, a metade dos pacientes com lesōes associadas da presente série era portadora de aneurisma do ventrículo esquerdo, com importante repercussão hemodinâmica e que requereu correção concomitante. Neste raciocínio também se incluem os casos de infarto do miocárdio de evoluçăo recente, os quais também apresentaram índices significativos de morbi-mortalidade, constituindo-se, porisso, num grupo de prognóstico cirúrgico reservado.

Um pequeno número de pacientes submeteu-se a 3a intervenção, e, afora as dificuldades inerentes, a obtenção de veia safena constituiu problema adicional, em alguns casos. Contudo, a aplicação das mamárias e veias cefálicas proporcionou revascularizaçāo satisfatória. Do ponto de vista de morbi-mortalidade, este subgrupo mostrou resultados favoráveis.

A angioplastia tem sido uma alternativa atrativa para pacientes previamente submetidos a revascularizaçāo cirúrgica do miocárdio ${ }^{8}$. O sucesso deste método é conseguido quando o diâmetro da estenose é reduzido a $20 \%$, ou menos, sem que ocorram complicações durante o procedimento, tais como óbito, reintervenção cirúrgica, infarto do miocárdio ${ }^{13}$. ERNST et alii ${ }^{8}$ relataram maior sucesso na dilatação das pontes, do que propriamente nas artérias nativas, na proporção de $97 \%$ versus $86 \%$ e consideram que a "idade" do enxerto não influenciou os resultados angiográficos sem que tenham ocorrido embolizaçōes distais originárias do ateroma do enxerto. $\mathrm{Na}$ sua casuística, de 83 pacientes submetidos a angioplastia, 11 foram encaminhados a cirurgia, 3 devido ao insucesso da dilatação e 8 pela recurrência da estenose. 
DINKHUYSEN, J. J.; SOUZA, L. C. B.; ANIJAR, A. M.; PAULISTA, P. P.; CHACCUR, P.; PIEGAS, L. S.; MANRIQUE, R.; ARNONI, A. S.; SOUSA, J. E. M. R.; JATENE, A. D. - O espectro da reoperação em cirurgia de coronária. Rev. Bras. Cir. Cardiovasc., $4(1): 32-42,1989$.

As indicaçōes para cirurgia, desde que eletivas, seguem a mesma metodologia dos pacientes candidatos a reoperação. Entretanto, quando o caso tem indicação cirúrgica de emergência, devido a complicaçōes da angioplastia, o prognóstico cirúrgico poderá ser afetado, tendo em vista a exigüidade de témpo disponivel diante das dificuldades técnicas das reoperações. Na nossa casuística, este subgrupo de pacientes submetidos a angioplastia não mostrou indices significativos de morbi-mortalidade.

Recentes publicaçōes ${ }^{6,9,17}$ dão conta de que a faixa etária constitui importante fator determinante de sobrevida precoce e tardia dos pacientes submetidos a reintervençōes coronárias. Casos com idade mais avançada, associados a função ventricular deteriorada, tendem a apresentar resultados menos favoráveis. FOSTER et alii $^{9}$ afirmam que pacientes mais jovens, com doença aterosclerótica pouco extensa, melhor função ventricular e ausência de insuficiência cardiaca, têm melhor perspectiva. Todavia, na nossa casuística, os pacientes mais jovens (entre 35 e 49 anos) mostraram menor morbidade e maior mortalidade da série. Poder-se-ia especular dizendo que os pacientes jovens suportam com mais vigor a operação e o pós-operatório, apresentando, quase que invariavelmente, uma recuperação mais precoce. Por outro lado, são portadores de doença aterosclerótica insidiosa, que compromete, mais precoce e difusamente, a circulação coronária e a função do ventrículo esquerdo, sem que ocorra formação de circulaçāo colateral intercoronária, a despeito da ocorrência prévia de revascularização cirúrgica do miocárdio. Em contraposição, o subgrupo com idade acima de 65 anos, conforme a expectativa, mostrou índices mais elevados de morbidade e, paradoxalmente, menor mortalidade. Possivelmente, a seleção criteriosa dos pacientes, a presença de graus mais definidos de comprometimento da circulação coronária geralmente amparada por extensa rede de circulação colateral e, por isso, com a função ventricular eventualmente mais preservada, podem constituir os fatores que expliquem estes achados.

RBCCV $44205-75$

DINKHUYSEN, J. J.; SOUZA, L. C. B.; ANIJAR, A. M.; PAULISTA, P. P.; CHACCUR, P.; PIEGAS, L. S.; MANRIQUE, R.; ARNONI, A. S.; SOUSA, J. E. M. R.; JATENE, A. D. - The spectrum os re-operation in coronary surgery. Rev. Bras. Cir. Cardiovasc., 4(1): 32-42, 1989.

ABSTRACT: The authors present the results obtained in the first 30 day P.O. of 204 patients who underwent reoperations for coronary atherosclerosis. The interval between first and second operation varied from 1 month to 16.5 years. There was also two small groups of patients of third intervention and with associated cardiac lesions. The basic strategy consisted on anticoagulation, the aplication of the mammary arteries (one or two) whenever possible, and revascularization as complete as possible. Most patients showed uneventfull evolution and a quarter of them presented some kind of non-fatal complication with good response to specific therapeutic measures. The overall mortality was about $9 \%$. Patients with atherosclerotic lesions in native coronary circulation presented the greater morbidity, but in those with atherosclerotic lesions both in the grafts and coronary native circulation, the mortality was higher. Contrary to expectation, the younger group showed higher mortality, and the older group presented higher morbidity. The aplication of the IMA's on the reintervention does not add morbidity or mortality, but the concomitant correction of the associated cardiac pathologies represented a higher risk in the procedure. Recent myocardial infarction influenced also morbidity. The third intervention, failure transluminal angioplastic coronary dilatation and the presence of previous IMA does not exert deleterious impact on the morbidity or mortality.

DESCRIPTORS: myocardial revascularization, reoperation.

\section{REFERÊNCIAS BIBLIOGRÁFICAS}

1 ADAM, M.; GRISLER, G. F.; LAMBERT, C. J.; MITCHEL Jr., B. F. - Reoperation following clinical failure of aorta-to-coronary artery bypass vein grafts. Ann. Thorac. Surg., 14: 272-279, 1972.

2 ARNONI, A. S.; PAULISTA, P. P.; SOUZA, L. C. B.; SOUSA, J. E. M. R.; FICHINO, M. Z. S.; ANGRISANI NETO, S.; BONATELLI FILHO, L.; JATENE, A. D. - Reoperação em cirurgia de revascularização do miocárdio. Arq. Bras. Cardiol., 41: 317-322, 1983.
3 BURLINGAME, N. W.; BONCHEK, L. I.; VAZALES, B. E. - Left thoracotomy for reoperative coronary bypass. J. Thorac. Cardiovasc. Surg., 95: 508-510, 1988.

4 CAMERON, A.; KEMP Jr, H. G.; SHIMOMURA, S.; SANTILLI, E.; GREEN, G. E.; HUTCHINSON III, J. E.; MEKHJIAN, H. A. - Aortocoronary bypass surgery: a 7 year follow-up. Circulation, 60: (Supl. 1): 9-13, 1979.

5 CAMPEAU, L.; LESPÉRANCE, J.; HERMANN, J.; CORBARA, F.; GRONDIN, C. M.; BOURASSA, M. G. - 
DINKHUYSEN, J. J.; SOUZA, L. C. B.; ANIJAR, A. M.; PAULISTA, P. P.; CHACCUR, P.; PIEGAS, L. S.; MANRIQUE, R.; ARNONI, A. S.; SOUSA, J. E. M. R.; JATENE, A. D. - O espectro da reoperação em cirurgia de coronária. Rev. Bras. Cir. Cardiovasc., 4(1): 32-42, 1989.

Loss of the improvement of angina between 1 and 7 years after aortocoronary bypass surgery: correlation with changes in vein grafts and in coronary arteries. Circulation, 60: (Supl. 1): 1-5, 1979.

6 COSGROVE, D. M.; LOOP, F. D.; LYTLE, B. W.; GILL, C. C.; GOLDING, L. A. R.; GIBSON, C.; STEWART, R. W.; TAYLOR, P. C.; GOORMASTIC, M. - Predictors of reoperations after myocardial revascularization. J. Thorac. Cardiovasc. Surg., 92: 811-821, 1986.

7 DINKHUYSEN, J. J.; SOUZA, L. C. B.; FICHINO, M. Z. S.; CHACCUR, P.; ARNONI, A. S.; PIEGAS, L. S.; MAGALHĀES, H. M.; PAULISTA, P. P.; SOUSA. J. E. M. R.; JATENE, A. D. - Anastomose mamáriacoronária: análise de 2923 casos. Rev. Bras. Cir. Cardiovasc., 2: 7-21, 1987.

8 ERNST, S. M. P. C.; VAN DER FELTZ, T. A.; ASCOOP, C. A. P. L.; BAL, E. T.; VERMENDEN, F. E. E.; KNAEPEN, P. J.; VAN BOGERIJEN, L, VANDENBERG, E. J. M.; PLOKKER, H. W. T. - Percutaneous transluminal coronary angioplasty in patients with prior coronary artery bypass grafting. J. Thorac. Cardiovasc. Surg., 93: 268-275, 1987.

9 FOSTER, E. D.; FISHER, L. P.; KAISER, G. C.; MYERS, W. O. - Comparison of operative mortality and morbidity for initial and repeat coronary artery bypass grafting: the coronary artery surgery study (CASS) registry experience. Ann. Thorac. Surg., 38: 563-570, 1984.

10 GRONDIN, C. M.; POMAR, J. L.; HERBERT, Y,; BOSH, X.; SANTOS, J. M.; ENJALBERT, M.; CAMPEAN, L. - Reoperation in patients with patent atherosclerotic coronary vein grafts. J. Thorac. Cardiovasc. Surg., 87: 379-385, 1984.

11 JOHNSON, W. D.; HOFFMAN Jr., J. F.; FLEMMA, R. J.; TECTOR, A. J. - Secondary surgical procedure for myocardial revascularization. J. Thorac. Cardiovasc. Surg., 64: 523-527, 1972.

JONES, E. L.; CARVER, J. M.; KING III, S. B.; DOUGLAS, J. S.; BRADFORD, J. M.; BROWN, C. M.; BONE, D. K.; HATCHER Jr., C. R. - Clinical anatomic and functional descriptor influencing morbidity, survival and adequacy of revascularization following coronary bypass. Ann. Surg., 192: 390-402, 1980.

KENT, K.; BENTIROGLIO, L. G.; BLOCK, P. C. - Percutaneous transluminal coronary angioplasty: report of the National Heart, Lung and Blood Institute. Am. J. Cardiol., 49: 2011-2020, 1982.

KEON, W. J.; HEGGTVEIT, H. A.; LEDUC, J. - Perioperative myocardial infarction caused by atheroembolism. J. Thorac. Cardiovasc. Surg., 84: 849-855, 1982. B. W.; TAYLOR, P. C.; GOLDING, L. A. R.; GROVES, L. K. - Late clinical and arteriografic results in $\mathbf{5 0 0}$

coronary artery reoperations. J. Thorac. Cardiovasc. Surg., 81: 675-685, 1981.

LOOP, F. D.; LYTLE, B. W.; GILL, C. C.; GOLDING, L. A. R.; COSGROVE, D. M.; TAYLOR, P. C. - Trends in selection and results of coronary artery reoperations. Ann. Thorac. Surg., 36: 380-388, 1983.

LYTLE, B. W.; LOOP, F. D.; COSGROVE, D. M.; TAYLOR, P. C.; CROOSMASTIC, M.; PEPER, W.; GILL, C. C.; GOLDING, L. A. R.; STEWART, R. W. - Fifteen hundred coronary reoperations: results and determinants of early and late survival. J. Thorac. Cardiovasc. Surg., 93: 847-859, 1987.

18 MULLER, R. \& MUSIKIC, P. - Hemorheology in surgery: a review. Angiology, 38: 581-592, 1987.

19 NATIONAL INSTITUTES OF HEALTH CONSENSUS. Development Conference Statement - Coronary-artery bypass surgery: scientific and clinical aspects. $N$. Engl. J. Med., 304: 680-681, 1981.

20

NORWOOD, W. I.; COHN, L. H.; COLLINS Jr., J. J. Results of reoperations for recurrent angina pectoris. Ann. Thorac. Surg., 23: 9-13, 1977.

PINTO, I. M. F.; PIEGAS, L. S.; MATTOS, L. A. P.; TANAJURA, L. F. L.; EGITO, E. T.; ABDULMASSIH NETO, C.; ARNONI, A. S.; SOUZA, L. C. B.; JATENE, A. D.; SOUSA, J. E. M. R. - Reoperação em pacientes revascularizados. Rev. Bras. Cir. Cardiovasc., 2: 171-174, 1987.

REUL, G. J.; COOLEY, D. A.; OTT, D. A.; COELHO, D. A, CHAPA, L.; ETEROVIC, I. - Reoperation for recurrent coronary artery disease: causes, indications and results in 168 patients. Arch. Surg., 114: 1269-1275, 1979.

SCHAFF, H. V.; ORSZULAK, T. A.; GERSH, B. J.; PIEHLER, J. M.; PUGA, F. J.; DANIELSON, G. K.; PLUTH, J. R. - The morbidity and mortality of reoperation for coronary artery disease and analysis of late results with use of actuarial estimate of even-free interval. $J$. Thorac. Cardiovasc. Surg., 85: 508-515, 1983.

STILES, Q. R.; LINDESWITH, G. G.; TUCKER, B. L.; HUGHES, R. K.; MEYER, B. W. - Experience with fifty repeat procedures for myocardial revascularization. J. Thorac. Cardiovasc. Surg., 72: 849-851, 1976.

UNGERLEIDER, R. M.; MILLS, N. L.; WECHSLER, A. S. - Left thoracotomy for reoperative coronary artery bypass procedures. Ann. Thorac. Surg., 40: 11-15, 1985.

WALTS, A. E.; FISHBEIN, M. C.; SUSTAINS, H.; MATTLOFF, J. M. - Rupture atheromatous plaques in saphenous vein coronary artery bypass grafts: a mechanism of acute, thrombotic, late graft occlusion. Circulation, 65: 197-201, 1982. 
DINKHUYSEN, J. J.; SOUZA, L. C. B.; ANIJAR, A. M.; PAULISTA, P. P.; CHACCUR, P.; PIEGAS, L. S.; MANRIQUE, R.; ARNONI, A. S.; SOUSÁ, J. E. M. R.; JATENE, A. D. - O espectro da reoperaçāo em cirurgia de coronária. Rev. Bras. Cir. Cardiovasc., 4(1): 32-42, 1989.

\section{Discussão}

\section{DR. MIGUEL ANGEL MALUF} São Paulo, SP

Gostaríamos de agradecer à Comissão Organizadora a honra de podermos comentar tão importante trabalho e felicitar os autores pelo excelente material e resultados apresentados. Todos sabemos que a aterosclerose é uma doença progressiva que afeta tanto os vasos enxertados como os vasos nativos, pelo que as reoperaçōes em cirurgia de coronária têm sido uma constante nos Serviços de Cirurgia Cardíaca. Como foi citado no trabalho de Shark (Am. Heart J., 102: 303-307, 1981), é potencialmente perigoso empregar indiscriminadamente os conhecimentos decorrentes da cirurgia de revascularização por enxertos primários para as reintervençōes. Estes fatos foram muito bem colocados pelos autores, onde mostram que os candidatos a reoperaçōes apresentam angina mais incapacitante e grave, evoluindo em classe III ou IV da NYHA, e uma porcentagem alta de infarto agudo do miocárdio prévio à reinternação (132 pacientes/65\%). Conforme foi exposto na apresentação, todos os pacientes da série encontram-se no grupo denominado de "sintomáticos", ou seja a causa principal da indicação de reestudo e reoperaçāo foi a sintomatologia, precedida, ou năo, de IAM. Existe o chamado grupo "assintomático", onde a insuficiência coronária pós revascularização do miocárdio é um achado cineangiográfico, quando esses pacientes sāo estudados rotineiramente no pós-operatório. Esses achados são decorrentes de erros técnicos, pontes fechadas etc., e poderão ser submetidos a um procedimento angioplástico, ou reoperaçōes com quadro clínico estável, realizado em condiçōes técnicas satisfatórias. O trabalho de Koshal (The Canadian Journal of Surgery, 2: 173-177, 1980) mostrou que este último grupo constituiu 25 a $30 \%$ de sua casuística e apenas $22 \%$ dos pacientes foram operados com IAM ocorrido no intervalo das operaçōes e com mortalidade na reoperação de 1,3\%. Talvez este seja o caminho mais difícil, embora mais gratificante. Outro assunto que gostaria de salientar é o que faz referência ao tipo de enxerto a ser utilizado nas reoperaçōes. Sem lugar a dúvidas, o emprego das artérias mamárias internas é considerado como primeira opção, pelos resultados a longo prazo já conhecidos, sendo a veia safena interna a segunda alternativa. Na prática, essa associação permite realizar a reoperação em mais de $90 \%$ dos casos, porém um grupo pequeno de pacientes fica sem opção na escolha do enxerto. Na nossa experiência, os enxertos homólogos, heterólogos, tubos de PTFE (politetrafluoretileno) existentes no mercado, assim como o uso de veias dos membros superiores, nāo os consideramos confiáveis, pela alta incidência de obstrução. Uma paciente de nossa casuistica, safenectomizada bilateralmente, foi reoperada 3 anos depois da cirurgia primitiva, pela obstrução das anastomoses das duas artérias mamárias, sendo realizado um enxerto livre de artéria gas- tro-omental, com bom resultado. Essa alternativa foi escolhida baseada nos resultados da experiência clínica do nosso grupo, acreditando que poderá ser incluída dentro do elenco de opçōes para o cirurgião. O último ponto a ser discutido refere-se ao manuseio das pontes de veia safena com graus avançados de aterosclerose. Os autores descrevem uma sistemática lógica na técnica cirúrgica, com a finalidade de evitar a embolia coronária por restos de ateromas deslocados das pontes de safena. Concordamos com o fato de que a soluçāo cardioplégica possa ser o veículo responsável da embolização coronária por ateromas soltos das pontes de safena. Como já foi mencionado, a cardioplegia através do óstio coronário seria uma boa alternativa; porém não temos experiência com o método. Finalmente, gostaria de apresentar os resultados da nossa experiência, em um grupo de 130 pacientes submetidos a reoperação de coronária, entre janeiro de 1982 e março de 1989. Foram divididos em 2 grupos: o primeiro, constituído por 92 pacientes operados com o auxilio da circulaçāo extracorpórea e o segundo, constituído por 38 pacientes operados sem circulação extracorpórea. A média de idades foi semeIhante; o número de pontes foi pouco menor no $2{ }^{\circ}$ grupo, devido, talvez, à possibilidade de abordagem das artérias DA, diagonal e direita somente. O emprego da artéria mamária interna foi semelhante, em ambos os grupos. Como resultado final, no grupo operado com circulação extracorpórea, houve $5,4 \%$ de infarto agudo do miocárdio per-operatório e mortalidade de $10,8 \%$, contra $2,6 \%$ de infarto agudo do miocárdio, nāo havendo mortalidade no grupo operado sem circulação extracorpórea. Temos que acrescentar outras vantagens neste último grupo, como menor área de dissecção e sangramento, ausência das complicações indesejáveis do uso da circulação extracorpórea e tempo de permanência hospitalar mais curto.

DR. WALDIR JAZBIK

Rio de Janeiro, RJ

Antes de iniciar meus comentários, gostaria de parabenizar o Dr. Jarbas Dinkhuysen, pela escolha do tema: "O espectro da reoperação em cirurgia de coronária", o qual foi abordado de maneira completa e bem objetiva. Sem dúvida, a era das reoperações coronárias chegou, já que o êxito da cirurgia de revascularização primária, em termos de sobrevida precoce e tardia combinados com a natureza progressiva da doença aterosclerótica, produz um número cada vez maior de pacientes candidatos à 2:, 3: e 4 intervençāo cirúrgica para revascularização do miocárdio. A cirurgia de reoperaçāo coronária representa um desafio para o cirurgião, devido às suas características peculiares. Desse modo, a abordagem do paciente por um cirurgião experiente é muito importante. Ao analisarmos a técnica cirúrgica utilizada, observamos que o autor não disseca os vasos femorais previamente à toracotomia, para uma possível entrada rápida 
DINKHUYSEN, J. J.; SOUZA, L. C. B.; ANIJAR, A. M.; PAULISTA, P. P.; CHACCUR, P.; PIEGAS, L. S.; MANRIQUE, R.; ARNONI, A. S.; SOUSA, J. E. M. R.; JATENE, A. D. - O espectro da reoperaçāo em cirurgia de coronária. Rev. Bras. Cir. Cardiovasc., 4(1): 32-42, 1989.

em circulação extracorpórea, em caso de acidente durante a abertura do tórax. Essa técnica é empregada em todos os grandes centros internacionais, como medida de segurança, pois existe grande possibilidade de lesōes graves das estruturas cardiacas e vasculares. Reiteramos, também, o cuidado com os enxertos pérvios com doença aterosclerótića, pelo risco de embolização coronária. A heparinização precoce com controle do TCA é muito importante e merece os nossos elogios, pois, além de sua ação profilática para eventuais estados de hiperagregabilidade, facilita o reaproveitamento de sangue durante a dissecção cardiaca, diminuindo a necessidade de reposição no pós-operatório. Quanto à técnica de preservação miocárdica utilizando paradas anóxicas intermitentes com hipotermia moderada a $32^{\circ} \mathrm{C}$, somos desfavoráveis (em certos pacientes), devido ao grande risco de vários pinçamentos aórticos, poderem provocar a liberação de material aterosclerótico, causando complicaçōes embólicas. Damos preferência ao pinçamento coronário super-seletivo; quando esta técnica não é possível, utilizamos solução cardioplégica cristalóide com um único pinçamento aórtico associado a uma hipotermia sistêmica em torno de $28^{\circ} \mathrm{C}$. Somos de opinião que, nos pacientes com IAM recente, ou naqueles com síndrome isquêmica aguda, a cardioplegia cristalóide não deve ser utilizada. Não empregamos os enxertos pérvios como conduto para solução cardioplégica, pelo risco de embolização. A taxa de patenticidade encontrada pelo autor, para os enxertos de artéria mamária, não corresponde ao usual, porém sua análise ficou prejudicada, devido ao grande intervalo sem a utilização deste conduto. Sabemos que a artéria mamária interna apresenta um elevado índice de patenticidade, mesmo quando estudamos enxertos com mais de 12 anos, sendo o seu comportamento muito superior aos enxertos venosos. Utilizamos, sempre que possível, a artéria mamária interna unilateral, na cirurgia de revascularização primária e nos casos de reoperação. Concordamos com a necessidade de ressecçāo ou ligadura das pontes de safena comprometidas pela aterosclerose como profilaxia da embolização; porém, utilizamos, quando possivel, a porção distal (justa coronária) do enxerto para realizar a nova anastomose na coronária, assim como a porção proximal (aórtica), para facilitar a anastomose do novo enxerto na aorta. A mortalidade geral apresentada pelos autores encontra-se entre os níveis alcançados nos diversos centros, podendo atingir cifras inferiores. Porém, quando a mortalidade é relacionada com a idade, observamos uma taxa paradoxal. Não observamos, no trabalho nem na literatura, atenção especial quanto ao preparo do enxerto venoso. Analisando o princípio da oclusão dos enxertos venosos, poderemos determinar estes cuidados. Basicamente, existem 2 tipos de lesōes oclusivas do enxerto venoso: a hiperplasia fibrótica da íntima e a aterosclerose. Observamos que enxertos de pacientes hipertensos apresentam maior grau de hiperplasia fibrótica, enquanto que, nos pacientes portadores de dislipidemias, a aterosclerose é mais intensa. Ambos os tipos de lesōes podem estar associados, ou não, à trombose.
Acreditamos que estas lesōes, embora diferentes quando analisadas do ponto de vista anatomopatológico, tenham como origem comum uma lesão primária da íntima. Esta lesão primária poderia ser espontânea, como na hipertensão arterial, ou provocada quando do manuseio da veia, através da aplicação de grande pressão com soluçōes cristalóides (ditas isotônicas), na hora de seu preparo. Como podemos explicar o fato de encontrarmos, em um paciente, os dois tipos de lesōes? Um enxerto com predomínio de hiperplasia fibrótica da íntima e outro enxerto com aterosclerose difusa bem desenvolvida em pacientes normotensos. Acreditamos que a resposta para esta questão esteja em um melhor preparo do enxerto, utilizando cuidados específicos, tais como: preparo da veia com sangue em baixa pressão. Gostaria de agradecer à Comissão Organizadora deste Congresso, pela oportunidade de comentar o trabalho do $\mathrm{Dr}$. Dinkhuysen.

\section{DR. DINKHUYSEN \\ (Encerrando)}

Agradeço os comentários feitos pelos Drs. Maluf e Jazbik. Sem dúvida, este grupo de pacientes tem por característica fundamental ser portador de formas insidiosas de aterosclerose, que podem comprometer, não somente a circulação coronária nativa, mas também os enxertos confeccionados previamente, requerendo, por isto, cuidados redobrados por parte da equipe cirúrgica. Chamo a atenção para que foram incluídos nesta casuística pacientes com lesões associadas que foram concomitantemente tratadas. De um modo geral, as publicaçōes têm demonstrado resultados em grupos cirúrgicos nos quais apenas foram tratadas as coronárias, conforme mostrou o Dr. Maluf. Uma das conclusões deste trabalho refere-se à maior mortalidade observada neste grupo específico e que influenciou, decisivamente, nos resultados finais. A aplicação de enxertos alternativos, principalmente nas reoperações de coronárias naqueles casos em que não se dispōe de veias safenas e das artérias mamárias, pode se constituir, muitas vezes, em um dilema. Tive a oportunidade de acompanhar uma revascularização coronária com artéria gastro-omental, ocasião na qual o resultado foi bem satisfatório; contudo, acho que seu emprego deve ser relegado a casos muito selecionados, não sendo uma técnica facilmente reprodutível e de aplicação indiscriminada. A contribuição que o grupo da Escola Paulista de Medicina tem prestado à cirurgia cardiaca brasileira é vigorosa e um dos seus maiores pontos constitui-se na revascularização do miocárdio sem emprego da circulação extracorpórea. No seu comentário, o Dr. Maluf chama a atenção para 2 grupos de pacientes que foram para reoperação, $1 \mathrm{com}$ auxilio da circulação extracorpórea e outro sem, sendo que, no primeiro, a ocorrência de IAM de óbitos foi signifi-. cativamente superior. Contudo, deve ser ressaltado que o grupo sem circulação extracorpórea é menor e realizou 
DINKHUYSEN, J.J.; SOUZA, L. C. B.; ANIJAR, A. M.; PAULISTA, P. P.; CHACCUR, P.; PIEGAS, L. S.; MANRIQUE, R.; ARNONI, A. S.; SOUSA, J. E. M. R.; JATENE, A. D. - O espectro da reoperaçāo em cirurgia de coronária. Rev. Bras. Cir. Cardiovasc., 4(1): $32-42,1989$.

menor número de pontes por paciente, possivelmente pela limitação de abordagem das artérias coronárias que o método eventualmente apresenta. Este fato provoca, necessariamente, uma seleção bem mais detalhada dos pacientes, fazendo com que os resultados finais sejam mais favoráveis, quando comparados com o grupo que usou circulaçāo extracorpórea. Ao Dr. Jazbik, diria que reoperaçōes coronárias săo relativamente freqüentes, em nosso Serviço, e, realmente, os cirurgiōes não esperam acidentes na toracotomia. Neste grupo de pacientes, tivemos apenas 1 caso que requereu manobras especiais, dada a presença de um aneurisma de aorta ascendente que se rompeu na abertura do tórax e que foi reparado sem maiores problemas ao paciente. Quanto à heparinização prévia com controle do TCA, notadamente naqueles pacientes que apresentam pontes de safena com lesōes obstrutivas, a nossa impressão é de que dá mais segurança ao procedimento, evitando estados de hiperagregabilidade. Por outro lado, não temos observado sangramento excessivo, durante a tora- cotomia. A rotina, em nosso Serviço no Hospital do Coração, é operar os coronarianos e as reoperaçōes de coronárias com pinçamento intermitente da aorta em hipotermia sistêmica de $32^{\circ} \mathrm{C}$, já há décadas. Em raras ocasiōes, a cardioplegia cristalóide foi usada e, sem dúvida, constitui-se em um excelente método de proteção miocárdica. Contudo, é possível especular-se dizendo que a cardioplegia tem menor densidade e é mais fluída. Ao ser infundida com pressão na raiz da aorta, pode ser possivel que provoque o desprendimento de material aterosclerótico das pontes de safena, provocando, assim, embolização periférica com graves conseqüências. O sangue, por sua vez, também tem possibilidade teórica de provocar essa embolização durante as aberturas da pinça de aorta. O certo é que ambos os métodos têm seus defensores e discutir este tópico, no momento, é inapropriado, dada a exigüidade de tempo de que disponho nos comentários finais. Finalizando, aproveito a oportunidade para agradecer à Comissāo Organizadora deste Congresso o privilégio de apresentar este trabalho. 